\title{
Pancreatic ductal adenocarcinoma: time for a neoadjuvant revolution?
}

\author{
Roberto Salvia $^{1} \cdot$ Giuseppe Malleo $^{1} \cdot$ Laura Maggino $^{1} \cdot$ Michele Milella $^{2} \cdot$ Claudio Bassi $^{1}$
}

Published online: 22 May 2020

(c) Italian Society of Surgery (SIC) 2020

In his landmark publication "The structure of scientific revolutions" Harvard Philosopher Thomas Kuhn challenged the traditional view of science as the progressive accumulation of knowledge, and affirmed the concept of "progress through revolutions". In this framework, the reiteration of anomalies within a given conceptual network triggers a crisis whereby the underlying assumptions of the field are reexamined and a new paradigm is established. Maybe for the first time in the management of pancreatic ductal adenocarcinoma (PDAC), we are standing on the edge of such revolutionary shift.

The traditional paradigm of PDAC treatment is condensed in the well-known adage: "Surgery represents the only chance of cure for PDAC", which marks the introduction of most surgical research papers of the last decades. Given an aggressive malignancy diagnosed at an early stage so that surgery is feasible, would it not sound somewhat foolish not to resect it as soon as possible? Yet, this surgery-first approach recently began to falter. A deeper understanding of the biological nature of PDAC led to envision systemic spreading as an inherent feature of the tumor very early in its natural history. This is in line with the clinical observation that around $20 \%$ of the patients experience recurrence within six months, $40 \%$ within the first year after surgery, even in the case of a margin-free resection [1]. In this light surgery, albeit extensive, has no means to ensure a proper tumor clearance, advocating for a multimodal treatment approach. However, an increasing body of pragmatic evidence demonstrated that postoperative morbidity, performance status deterioration, and immediate recurrence might impair access to adjuvant therapy in up to one third of resected patients,

Roberto Salvia

Roberto.salvia@univr.it

1 Unit of General and Pancreatic Surgery, Department of Surgery, Dentistry, Pediatrics and Gynecology, University of Verona Hospital Trust, P.Le L.A, Scuro 10, 37134 Verona, Italy

2 Department of Medicine, Section of Medical Oncology, University of Verona Hospital Trust, Verona, Italy hindering the delivery of this-yet crucial—multimodality [2].

Newly introduced chemotherapy regimens, such as FOLFIRINOX and Gemcitabine plus Nab-paclitaxel recently exhibited favorable results in advanced settings [3, 4], granting a proportion of locally advanced (LA) and even metastatic patients with surgical opportunity, with resection rates ranging between $0-40 \%$ for LA [4] and $4-9 \%$ for initially metastatic PDAC [5]. This fostered interest in their possible implementation within a neoadjuvant treatment (NAT) framework. According to the National Cancer Institute Dictionary, NAT is "a treatment given as a first step to shrink a tumor before the main treatment, which is usually surgery, is given" so that this term should be rightfully referred only to resectable (R) or borderline resectable (BR) patients. The putative advantages of NAT are multifold. Its primary aim is to downstage and/or downsize the tumor, increasing the likelihood of a margin-free resection. Moreover, NAT could be theoretically offered to a greater number of patients relative to adjuvant therapy because of its early administration in subjects with a better performance status than those who have received resection. Another possible benefit of NAT is the timely treatment of radiologically occult micrometastases. Its administration is also thought to exert a selection effect by enucleating patients with either aggressive tumor biology or low physiologic resilience, and spare them the risks of a highly morbid operation.

Level I evidence comparing the outcomes of NAT with upfront surgery for R and BR patients is limited, with only three fully published manuscripts available [6-8]. Nonetheless, results appear encouraging, with slightly lower resection rates but longer survival after NAT (Table 1). While the full results of ongoing trials [9] are eagerly awaited, the steady uptake of NAT is already shifting the paradigm of PDAC management. At our Institution, the proportion of patients receiving chemotherapy \pm radiation prior to pancreatectomy has risen from $<15 \%$ before 2013 to $>50 \%$ in 2019. Notably, more than one third of these have a radiologically resectable disease at diagnosis. 


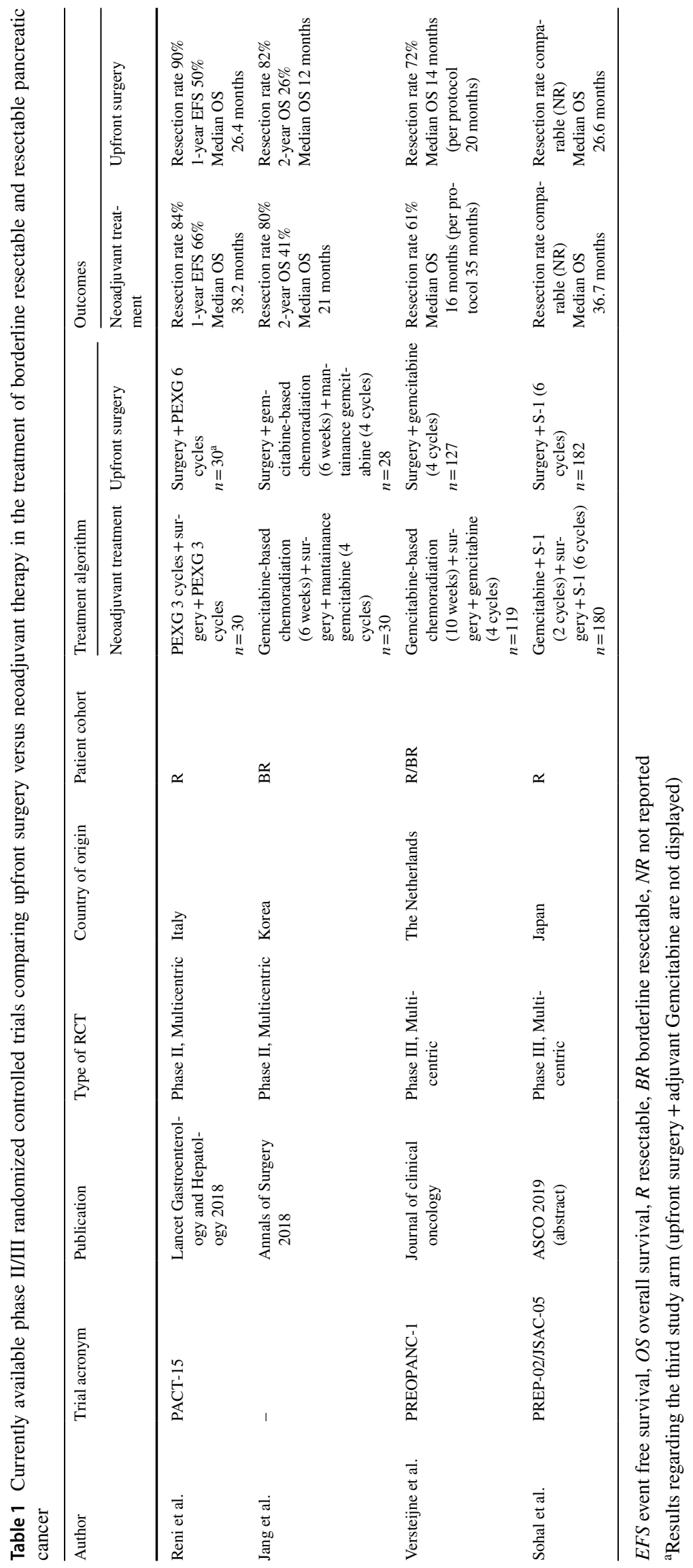


That considered, should a neoadjuvant-always approach become the new standard of care for PDAC? Indeed, evidence is not mature yet to answer this question, and the assumptions regarding the possible advantages of NAT remain largely speculative. Most of our knowledge is derived from surgical series, where the denominator is represented by the bulk of surgically explored patients and not by the total number of PDAC diagnoses. Consequently, there is little information on patients who were recommended NAT with intent for later resection but did not ultimately receive surgery (e.g., due to disease progression or decline in performance status during treatment). In a recent observational analysis of $614 \mathrm{LA}$ and BR patients who were recommended primary chemotherapy at our Institution between 2013-2015, only $93 \%$ actually received and $72 \%$ completed the treatment. In this real-life snapshot, the resection rate was $15 \%$ in the overall cohort, rising up to $53 \%$ in the subgroup of younger patients with anatomically BR disease who completed FOLFIRINOX [10]. Whether this should be looked at as a positive selection effect-as patients who progressed or declined during NAT would have experienced early recurrence or impaired access to adjuvant therapy after upfront surgery-is impossible to ascertain. Moreover, complications associated with cyto-histological confirmation and/or biliary decompression before or during NAT might significantly hinder surgical eligibility. Other controversial issues regard the preferable treatment regimens and the role of complementary radiation therapy. Also the optimal duration and the selection criteria for surgery after NAT remain undisclosed, with significant variability between institutions. Notably, these controversies can hardly be solved only relying on randomized evidence. In real-world conditions, a sizable amount of patients are elderly or present with certain conditions (e.g., jaundice, comorbidities, deteriorated performance status) that may preclude treatment eligibility and enrollment in research protocols. This is testified by the extremely low enrollment rate of current trials of NAT [7, 8], suggesting strict patients selection and questioning the degree to which their results can be translated to the everyday practice. Well-designed observational studies appear of great importance to acquire pragmatic information on the effects of NAT at the population level.

In the end, although at the Verona Pancreas Institute we do embrace the current neoadjuvant revolution, this is not with blind enthusiasm. While the majority of patients harbor a systemic disease already at the time of diagnosis and would benefit from receiving systemic treatment first-line, there likely are a number of subjects who might have a different disease biology and would gain more advantage from an upfront resection. How to segregate the two scenarios remains however undisclosed. As per international guidelines [11], we currently recommend NAT to all BR PDAC and also to $\mathrm{R}$ patients exhibiting risk features such as high
Ca19.9 serum levels, tumor contact with major vessels, suspect of metastases or distant lymph node involvement, and/or poor performance status. Yet, we must acknowledge that from a biological standpoint the value of classifications based on the degree of vascular involvement is almost nil. This is even truer considering that vascular resections-at least involving major veins - can now be performed with acceptable morbidity. In this setting, adopting different treatment modalities for R and BR is at best unwarranted.

If treatment allocation is currently mainly based on clinical, biochemical, and radiologic features, looking forward the nearest challenge appears to be the identification of new tools to better predict tumor response and develop personalized patient management pathways. Novel biomarkers such as circulating tumor cells, DNA and exosomes are currently scrutinized and their clinical implementation is forthcoming. PDAC management is moving from a radiology-driven to a biology-driven decision-making, whereby the treatment strategy and the degree of surgical aggressiveness will not anymore be based on anatomical classification but tailored to each tumor's specific genetic and molecular characteristics. Paradoxically, in the current era of extreme surgical proficiency, whereby technically almost anything is possible, our goal as pancreatic surgeons appears not to resect the largest number of patients, but to improve the identification of those who will truly benefit from our intervention. In this selected group, we should have the courage to push our resection beyond the borders of our best surgical technique. In these times of revolutions, let us envision two new surgical hashtags: \#SelectBetter, \#BeBraveAfterChemotherapy.

Funding None.

\section{Compliance with ethical standards}

Disclosure of potential conflicts of interest None.

Research involving Human Participants and/or Animals This article does not contain any studies with human participants performed by any of the authors.

Informed consent For this type of study formal consent is not required.

\section{References}

1. Groot VP, Gemenetzis G, Blair AB (2019) Defining and predicting early recurrence in 957 patients with resected pancreatic ductal adenocarcinoma. Ann Surg 269(6):1154-1162. https://doi. org/10.1097/SLA.0000000000002734

2. Mackay TM, Smits FJ, Roos D et al (2019) The risk of not receiving adjuvant chemotherapy after resection of pancreatic ductal 
adenocarcinoma: a nationwide analysis. HPB (Oxford). https:// doi.org/10.1016/j.hpb.2019.06.019

3. Von Hoff DD, Ervin T, Arena FP et al (2013) Increased survival in pancreatic cancer with nab-paclitaxel plus gemcitabine. N Engl J Med 369:1691-1703

4. Suker M, Beumer BR, Sadot E et al (2016) FOLFIRINOX for locally advanced pancreatic cancer: a systematic review and patient-level meta-analysis. Lancet Oncol 17:801-810

5. Crippa S, Cirocchi R, Weiss MJ et al (2020) A systematic review of surgical resection of liver-only synchronous metastases from pancreatic cancer in the era of multiagent chemotherapy. Updates Surg. https://doi.org/10.1007/s13304-020-00710-z

6. Jang J-Y, Han Y, Lee H et al (2018) Oncological benefits of neoadjuvant chemoradiation with gemcitabine versus upfront surgery in patients with borderline resectable pancreatic cancer: a prospective, randomized, open-label, multicenter phase $2 / 3$ trial. Ann Surg 268:215-222

7. Reni M, Balzano G, Zanon S et al (2018) Safety and efficacy of preoperative or postoperative chemotherapy for resectable pancreatic adenocarcinoma (PACT-15): a randomised, open-label, phase 2-3 trial. Lancet Gastroenterol Hepatol 3:413-423
8. Versteijne E, Suker M, Groothuis K et al (2020) Preoperative chemoradiotherapy versus immediate surgery for resectable and borderline resectable pancreatic cancer: results of the Dutch Randomized Phase III PREOPANC Trial. J Clin Oncol. https://doi. org/10.1200/JCO.19.02274(Epub ahead of print)

9. Salvia R, Casciani F, Sereni E, Bassi C (2019) Pancreatic cancer-What's next? Presse Med 48(3 Pt 2):e187-e197. https://doi. org/10.1016/j.lpm.2019.02.031

10. Maggino L, Malleo G, Marchegiani G et al (2019) Outcomes of primary chemotherapy for borderline resectable and locally advanced pancreatic ductal adenocarcinoma. JAMA Surg. https ://doi.org/10.1001/jamasurg.2019.2277 (Epub ahead of print)

11. NCCN clinical practice guidelines in oncology. Pancreatic adenocarcinoma. National Comprehensive Cancer Network. 2020 version 1

Publisher's Note Springer Nature remains neutral with regard to jurisdictional claims in published maps and institutional affiliations. 